\title{
Biometric viscera and blood parameters of meat quails supplemented with inorganic selenium and vitamin $\mathbf{E}$
}

\author{
Biometria das vísceras e parâmetros sanguíneos de codornas de corte suplementadas \\ com selênio inorgânico e vitamina $E$
}

\author{
ZANCANELA, Vittor ${ }^{1,2}$; FURLAN, Antonio Claudio ${ }^{2}$; POZZA, Paulo César ${ }^{2}$; \\ MARCATO, Simara Márcia ${ }^{2}$; GRIESER, Daiane de Oliveira ${ }^{2}$; STANQUEVIS, \\ Caroline Espejo ${ }^{2}$; FINCO, Eline ${ }^{2}$; FERREIRA, Mariana de Fátima Zanon ${ }^{3}$; OLIVEIRA- \\ BRUXEL, Taciana Maria de ${ }^{4}$
}

\footnotetext{
${ }^{1}$ Universidade Federal de Sergipe, Núcleo de Zootecnia, Nossa Senhora da Glória, Sergipe, Brasil.

${ }^{2}$ Universidade Estadual de Maringá, Departamento de Zootecnia, Maringá, Paraná, Brasil.

${ }^{3}$ JBS Foods, Rolândia, Paraná, Brasil.

${ }^{4}$ Universidade Estadual do Oeste do Paraná, Departamento de Zootecnia, Marechal Cândido Rondon, Paraná, Brasil.

*Endereço para correspondência: vitorzoo@hotmail.com
}

\section{SUMMARY}

This study aimed to evaluate biometric viscera and blood parameters of quails at 14 and 35 days of age, supplemented with different levels of inorganic selenium and vitamin E. A completely randomized design was used in a $4 \times 4$ factorial scheme (inorganic $\mathrm{Se}=0.1125$, $0.2250,0.3375$ and $0.4500 \mathrm{mg} / \mathrm{kg}$ diet $\mathrm{x} \mathrm{VE}=$ $10,23,36$ and $49 \mathrm{IU} / \mathrm{kg}$ feed). In the $0-14$ days experiment, 2,400 newborn quail were used, distributed in 16 treatments and three replications of 50 birds each. In the 14-35 days experiment, 1,680 14 day-old quails were used with same treatments and three replicates of 35 birds each. At 14 days, the relative spleen weights $(\mathrm{P}=0.0203)$ increased linearly as a function of the VE level, while at 35 days, the relative weight of the bursa $(\mathrm{P}=0.0390)$ increased linearly as a function of $\mathrm{Se}$ concentration. At 14 days, there was a vitamin $\mathrm{x}$ mineral interaction $(p=0.0097)$ affecting total cholesterol (TC), and a quadratic effect $(\mathrm{P}=$ $0.0138)$ related to $\mathrm{Se}$ and linear reduction levels $(\mathrm{P}=0.0275)$ as a function of VE. At 35 days, the TC concentrations $(\mathrm{P}=0.0055)$ and triglycerides ( $\mathrm{TG}, \mathrm{P}=0.0220$ ) showed a quadratic effect for Se. The linear increase in spleen weight and bursa after 14 and 35 days respectively suggests an improved immune response as a function of VE and Se supplementation. To achieve the lowest concentration of TC and TG at 35 days of age, supplementation of 0.26 and 0.29 $\mathrm{mg} / \mathrm{Se} / \mathrm{kg} /$ feed, respectively, is recommended.

Keywords: alpha-tocopherol, Coturnix coturnix coturnix, enzymes, trace minerals

\section{RESUMO}

Objetivou-se avaliar a biometria das vísceras e os parâmetros sanguíneos de codornas de corte aos 14 e 35 dias de idade, suplementadas com diferentes níveis de selênio inorgânico e vitamina E. Utilizou-se um delineamento inteiramente casualizado, em esquema fatorial $4 \times 4$ (Se inorgânico $=0,1125 ; 0,2250 ; 0,3375$ e $0,4500 \mathrm{mg} / \mathrm{kg}$ de ração $\mathrm{x} \mathrm{VE}=10 ; 23 ; 36$ e 49 $\mathrm{UI} / \mathrm{kg}$ de ração). No experimento de 0 a 14 dias, foram utilizadas 2.400 codornas recémnascidas, distribuídas em 16 tratamentos e três repetições de 50 aves cada. No experimento de 14 a 35 dias, sob os mesmos tratamentos, foram utilizadas 1.680 codornas com 14 dias de idade e três repetições de 35 aves cada. Aos 14 dias, o pesos relativo de baço $(\mathrm{P}=0,0203)$ aumentou linearmente em função dos níveis de VE, enquanto aos 35 dias, o peso relativo de bolsa cloacal $(\mathrm{P}=0,0390)$ aumentou linearmente em função do Se. Aos 14 dias, verificou-se interação $(\mathrm{P}=0,0097)$ vitamínica $\mathrm{x}$ mineral nas 
concentrações de colesterol total (CT), além de efeito quadrático $(\mathrm{P}=0,0138)$ relacionado aos níveis de Se e redução linear $(\mathrm{P}=0,0275)$ em função da VE. Aos 35 dias, as concentrações de CT $(\mathrm{P}=0,0055)$ e triglicerídeos (TGR, $\mathrm{P}=0,0220)$ apresentaram efeito quadrático para o Se. O aumento linear do peso do baço e bolsa cloacal aos 14 e 35 dias respectivamente, sugerem melhora na resposta imune em função da suplementação de VE e Se. Para a mínima concentração de CT e TGR aos 35 dias de idade, recomenda-se a suplementação de 0,26 e $0,29 \mathrm{mg} / \mathrm{Se} / \mathrm{kg} /$ ração respectivamente.

Palavras-chave: alfa-tocoferol, Coturnix coturnix coturnix, enzimas, microminerais

\section{INTRODUCTION}

The micromineral selenium can be supplied in the diet of birds in organic or inorganic form, the latter being traditionally more used. Their requirements are low, but if they are not met, the antioxidant system may be compromised, causing serious consequences for the animal's metabolism (SURAI et al., 2006).

Vitamin E plays several roles in the body, affecting meat quality, cellular respiration and nucleic acid metabolism, and also acting as a potent antioxidant for unsaturated fatty acids and vitamin A (TOLEDO et al., 2006).

Suhajda et al. (2000) reported that since the association of selenium with vitamin E was established, along with the modulating effect of glutathione peroxidase and antioxidants on the actions of free radicals and peroxides, the investigation of these elements has intensified.

Determination of biochemical parameters in the blood is a tool that assists in the diagnosis of metabolic diseases, contributes to the definition of the nutritional profile of a population, and also allows a more detailed clinical evaluation of a population of individuals (VALLE et al., 2008). According to Thrall et al. (2004), these parameters can be altered according to the nutritional status and also the age of the studied animal. Together with these parameters, evaluation of the relative weights of viscera and lymphoid organs can be used to indicate the deficiency or toxicity of particular elements (NRC, 1994) and also to assess how well the immune system is functioning (HABIBIAN et al., 2013).

Several studies report the effects of selenium and/or vitamin $\mathrm{E}$ on the performance and carcass yield (ŠEVČÍKOVÁ et al., 2006; SOUZA et al., 2006; HABIBIAN et al., 2016), meat quality (SOUZA et al., 2006; De ALMEIDA et al., 2012; CHEN et al., 2013) and even on blood parameters (ARSLAN et al., 2001; HABIBIAN et al., 2013) and viscera biometry (ALBUQUERQUE et al., 2017) of birds. However, little is known about the effects exerted by different levels of supplementation of these elements in quails, since most of the works found in the literature were conducted with broilers and evaluated the microingredients separately.

Based on these considerations, the objective of this work was to evaluate the effects of different levels of inorganic selenium and vitamin $\mathrm{E}$ supplementation using biometry of the viscera and blood parameters of meat quails at 14 and 35 days of age.

\section{MATERIAL AND METHODS}

The experiment was carried out in the Coturniculture sector of the Experimental Farm of Iguatemi, belonging to the State University of Maringá (UEM). The study was divided into the initial phase ( 0 to 14 days of 
age) and the final growth phase (15 to 35 days of age) of the meat quails, also known as European quails (Coturnix coturnix coturnix). The experiment was conducted according to the standards of the UEM Committee on Animal Experimentation (Protocol $\mathrm{n}^{\mathrm{o}}$ 079/2014).

Birds were housed in a shed with boxes of $2.5 \mathrm{~m}^{2}$, with a dirt floor and a bed of rice straw. The birds were divided into their respective treatment groups, using a completely randomized design, in a $4 \times 4$ factorial scheme (Inorganic $\mathrm{Se}=$ $0.1125, \quad 0.2250, \quad 0.3375$ and 0.4500 $\mathrm{mg} / \mathrm{kg}$ of feed $\mathrm{x} \mathrm{VE}=10,23,36$ and 49 $\mathrm{IU} / \mathrm{kg}$ of feed). In experiment 1 , from 0 to 14 days old, 2,400 newborn quails were used, distributed in 16 treatments with three replicates of 50 birds each. In experiment 2, from 14 to 35 days, 1,680 14 day-old quails were distributed into the same treatment groups, with three replicates of 35 birds each. The rations were formulated according to the recommendations of the Brazilian Tables for Poultry and Swine based on the food composition obtained by Rostagno et al. (2011). In the case of the maize and soybean meal used in this study, the composition was based on analyzes carried out at the Laboratory of Analysis and Animal Nutrition (LANA) at the State University of Maringá (UEM). The rations were formulated to meet the requirements of the birds at each age, except for Se and VE. To meet the requirements for available calcium and phosphorus in the diet, the values recommended by Silva et al. (2009) were used. The rations were adjusted to have the desired Se and VE levels as shown in Tables 1 and 2 (for the period from 0 to 14 days) and Tables 3 and 4 (for the period from 14 to 35 days). Inorganic Se was supplied as $45 \%$ sodium selenite and VE was supplied in the form of dl-tocopheryl acetate $(500,000 \mathrm{IU} / \mathrm{kg})$. Both were prediluted into mixtures, to reduce the possibility of dispersion and complexation of their particles during the feed manufacturing process. Each $100 \mathrm{~g}$ mixture contained the required level of the micro-ingredient for each treatment $(\mathrm{g})$ and was completed with inert materials (Caulin in the case of sodium selenite and rice straw in the case of vitamin E). Throughout the experimental period the birds received the ration and water ad libitum. The feed was supplied in tubular type feeders and water was supplied in pressure cup type drinking fountains ( 0 to 14 days) and bell drinkers (14 to 35 days) of age. No vaccine protocol was adopted for the birds.

For the evaluation of the blood parameters, four and two birds per experimental unit were slaughtered at 14 and 35 days of age, respectively for blood collection, after undergoing a five-hour feeding fast. The blood collection was performed by exsanguination, the samples being collected and conditioned in test tubes, and immediately centrifuged at 3,000 rpm for 15 minutes. The serum was separated and packed in polypropylene microtubes and stored at $-10^{\circ} \mathrm{C}$ until the analyses were performed. The total cholesterol -PP-MS-80022230064 (TC) and triglyceride -PP-MS 80022230062 (TG) concentrations and the activity of the enzymes aspartate aminotransferase PP-MS80022230083 (AST), alanine aminotransferase PP-MS 80022230086 (ALT), and creatine kinase -PP-MS 80022230088 (CK) were measured using a spectrophotometer (Model Bioplus 2000) in combination with commercial assay kits (Gold Analisa Diagnostica Ltda, Belo Horizonte MG). 
Table 1. Percent composition of experimental diet for growing meat quail ( 0 to 14 days old) supplemented with different levels of inorganic selenium and vitamin $\mathrm{E}$

\begin{tabular}{|c|c|c|c|c|c|c|c|c|c|c|c|c|c|c|c|c|}
\hline \multirow{2}{*}{$\begin{array}{l}\begin{array}{l}\text { Selenium } \\
(\mathrm{mg} / \mathrm{kg})\end{array} \\
\text { Vitamin E } \\
(\mathrm{IU} / \mathrm{kg})\end{array}$} & \multicolumn{4}{|c|}{0.1125} & \multicolumn{4}{|c|}{0.2250} & \multicolumn{4}{|c|}{0.3375} & \multicolumn{4}{|c|}{0.4500} \\
\hline & 10 & 23 & 36 & 49 & 10 & 23 & 36 & 49 & 10 & 23 & 36 & 49 & 10 & 23 & 36 & 49 \\
\hline Corn & 36.798 & 36.798 & 36.798 & 36.798 & 36.798 & 36.798 & 36.798 & 36.798 & 36.798 & 36.798 & 36.798 & 36.798 & 36.798 & 36.798 & 36.798 & 36.798 \\
\hline $\begin{array}{l}\text { Soybean meal } \\
45 \%\end{array}$ & 53.718 & 53.718 & 53.718 & 53.718 & 53.718 & 53.718 & 53.718 & 53.718 & 53.718 & 53.718 & 53.718 & 53.718 & 53.718 & 53.718 & 53.718 & 53.718 \\
\hline Soybean oil & 5.793 & 5.793 & 5.793 & 5.793 & 5.793 & 5.793 & 5.793 & 5.793 & 5.793 & 5.793 & 5.793 & 5.793 & 5.793 & 5.793 & 5.793 & 5.793 \\
\hline $\begin{array}{l}\text { Dicalciun } \\
\text { phosphate }\end{array}$ & 1.458 & 1.458 & 1.458 & 1.458 & 1.458 & 1.458 & 1.458 & 1.458 & 1.458 & 1.458 & 1.458 & 1.458 & 1.458 & 1.458 & 1.458 & 1.458 \\
\hline Limestone & 0.405 & 0.405 & 0.405 & 0.405 & 0.405 & 0.405 & 0.405 & 0.405 & 0.405 & 0.405 & 0.405 & 0.405 & 0.405 & 0.405 & 0.405 & 0.405 \\
\hline Dl-Methionine & 0.455 & 0.455 & 0.455 & 0.455 & 0.455 & 0.455 & 0.455 & 0.455 & 0.455 & 0.455 & 0.455 & 0.455 & 0.455 & 0.455 & 0.455 & 0.455 \\
\hline L-Lysine HCL & 0.196 & 0.196 & 0.196 & 0.196 & 0.196 & 0.196 & 0.196 & 0.196 & 0.196 & 0.196 & 0.196 & 0.196 & 0.196 & 0.196 & 0.196 & 0.196 \\
\hline L-Threonine & 0.109 & 0.109 & 0.109 & 0.109 & 0.109 & 0.109 & 0.109 & 0.109 & 0.109 & 0.109 & 0.109 & 0.109 & 0.109 & 0.109 & 0.109 & 0.109 \\
\hline Salt & 0.458 & 0.458 & 0.458 & 0.458 & 0.458 & 0.458 & 0.458 & 0.458 & 0.458 & 0.458 & 0.458 & 0.458 & 0.458 & 0.458 & 0.458 & 0.458 \\
\hline $\begin{array}{l}\text { Vit/min } \\
\text { suplement }{ }^{1}\end{array}$ & 0.400 & 0.400 & 0.400 & 0.400 & 0.400 & 0.400 & 0.400 & 0.400 & 0.400 & 0.400 & 0.400 & 0.400 & 0.400 & 0.400 & 0.400 & 0.400 \\
\hline $\begin{array}{l}\text { Selenite blend } \\
45 \%\end{array}$ & 0.100 & 0.100 & 0.100 & 0.100 & 0.100 & 0.100 & 0.100 & 0.100 & 0.100 & 0.100 & 0.100 & 0.100 & 0.100 & 0.100 & 0.100 & 0.100 \\
\hline Vitamin E blend & 0.100 & 0.100 & 0.100 & 0.100 & 0.100 & 0.100 & 0.100 & 0.100 & 0.100 & 0.100 & 0.100 & 0.100 & 0.100 & 0.100 & 0.100 & 0.100 \\
\hline Antioxidant $^{2}$ & 0.010 & 0.010 & 0.010 & 0.010 & 0.010 & 0.010 & 0.010 & 0.010 & 0.010 & 0.010 & 0.010 & 0.010 & 0.010 & 0.010 & 0.010 & 0.010 \\
\hline Total & 100 & 100 & 100 & 100 & 100 & 100 & 100 & 100 & 100 & 100 & 100 & 100 & 100 & 100 & 100 & 100 \\
\hline
\end{tabular}

${ }^{1}$ Vitamin/mineral supplementation free of selenium and vitamin E (guarantee levels per kg of product) Vit. A - 10.000.000 IU; Vit. D3 - 750.000 IU; Vit. B1 - 625 mg; Vit. B2 - 1,500 mg; Vit. B6 - 1250 mg; Vit. B12 - 5,000 mcg; Vit. K3 - 750 mg; Folic acid 250 mg/kg; Biotin 50 mg/kg; Calciun Pantothenate - 3,000 mg; Niacin - 6,000 mg; Choline Chloride - 75 g/kg; Zinc Oxide - 13 g/kg; Iron Sulfate - 12 g/kg; Manganese Sulfate - 15 g/kg; Cooper Sulfate - 2,500 mg; Cobalt Sulfate - $50 \mathrm{mg}$; Iodine - $250 \mathrm{mg}$; Selenium - 0 mg; BHT $1000 \mathrm{mg} / \mathrm{kg}$; Vehicle Q.S.P. (Caulin) $1.000 \mathrm{~g} / \mathrm{kg} .2$, and ${ }^{2}$ BHT (Butyl Hydroxy Toluene). 
Table 2. Nutritional composition of the experimental diets for growing meat quails ( 0 to 14 days old) supplemented with different levels of inorganic selenium and vitamin $\mathrm{E}$

\begin{tabular}{|c|c|c|c|c|c|c|c|c|c|c|c|c|c|c|c|c|}
\hline \multirow{2}{*}{$\frac{\text { Selenium (mg/kg) }}{\text { Vitamin E (IU/kg) }}$} & \multicolumn{4}{|c|}{0.1125} & \multicolumn{4}{|c|}{0.2250} & \multicolumn{4}{|c|}{0.3375} & \multicolumn{4}{|c|}{0.4500} \\
\hline & 10 & 23 & 36 & 49 & 10 & 23 & 36 & 49 & 10 & 23 & 36 & 49 & 10 & 23 & 36 & 49 \\
\hline Metabolizable energy (kcal/kg) & 2.997 & 2.997 & 2.997 & 2.997 & 2.997 & 2.997 & 2.997 & 2.997 & 2.997 & 2.997 & 2.997 & 2.997 & 2.997 & 2.997 & 2.997 & 2.997 \\
\hline Available phosphorus (\%) & 0.41 & 0.41 & 0.41 & 0.41 & 0.41 & 0.41 & 0.41 & 0.41 & 0.41 & 0.41 & 0.41 & 0.41 & 0.41 & 0.41 & 0.41 & 0.41 \\
\hline Calcium (\%) & 0.65 & 0.65 & 0.65 & 0.65 & 0.65 & 0.65 & 0.65 & 0.65 & 0.65 & 0.65 & 0.65 & 0.65 & 0.65 & 0.65 & 0.65 & 0.65 \\
\hline Crude protein $(\%)$ & 27.5 & 27.5 & 27.5 & 27.5 & 27.5 & 27.5 & 27.5 & 27.5 & 27.5 & 27.5 & 27.5 & 27.5 & 27.5 & 27.5 & 27.5 & 27.5 \\
\hline Lysine digestible (\%) & 1.60 & 1.60 & 1.60 & 1.60 & 1.60 & 1.60 & 1.60 & 1.60 & 1.60 & 1.60 & 1.60 & 1.60 & 1.60 & 1.60 & 1.60 & 1.60 \\
\hline Met.+cyst. digestible (\%) & 1.15 & 1.15 & 1.15 & 1.15 & 1.15 & 1.15 & 1.15 & 1.15 & 1.15 & 1.15 & 1.15 & 1.15 & 1.15 & 1.15 & 1.15 & 1.15 \\
\hline Threonine digestible (\%) & 1.04 & 1.04 & 1.04 & 1.04 & 1.04 & 1.04 & 1.04 & 1.04 & 1.04 & 1.04 & 1.04 & 1.04 & 1.04 & 1.04 & 1.04 & 1.04 \\
\hline Tryptophan digestible (\%) & 0.33 & 0.33 & 0.33 & 0.33 & 0.33 & 0.33 & 0.33 & 0.33 & 0.33 & 0.33 & 0.33 & 0.33 & 0.33 & 0.33 & 0.33 & 0.33 \\
\hline Chlorine (\%) & 0.32 & 0.32 & 0.32 & 0.32 & 0.32 & 0.32 & 0.32 & 0.32 & 0.32 & 0.32 & 0.32 & 0.32 & 0.32 & 0.32 & 0.32 & 0.32 \\
\hline Sodium (\%) & 0.20 & 0.20 & 0.20 & 0.20 & 0.20 & 0.20 & 0.20 & 0.20 & 0.20 & 0.20 & 0.20 & 0.20 & 0.20 & 0.20 & 0.20 & 0.20 \\
\hline Potassium (\%) & 1.09 & 1.09 & 1.09 & 1.09 & 1.09 & 1.09 & 1.09 & 1.09 & 1.09 & 1.09 & 1.09 & 1.09 & 1.09 & 1.09 & 1.09 & 1.09 \\
\hline Electrol. balance $\mathrm{mEq} / \mathrm{kg}$ & 275.02 & 275.02 & 275.02 & 275.02 & 275.02 & 275.02 & 275.02 & 275.02 & 275.02 & 275.02 & 275.02 & 275.02 & 275.02 & 275.02 & 275.02 & 275.02 \\
\hline
\end{tabular}


Table 3. Percent composition of experimental diet for growing meat quail (14 to 35 days old) supplemented with different levels of inorganic selenium and vitamin $\mathrm{E}$

\begin{tabular}{|c|c|c|c|c|c|c|c|c|c|c|c|c|c|c|c|c|}
\hline \multirow{2}{*}{$\begin{array}{l}\begin{array}{l}\text { Selenium } \\
(\mathrm{mg} / \mathrm{kg})\end{array} \\
\text { Vitamin E } \\
(\mathrm{IU} / \mathrm{kg})\end{array}$} & \multicolumn{4}{|c|}{0.1125} & \multicolumn{4}{|c|}{0.2250} & \multicolumn{4}{|c|}{0.3375} & \multicolumn{4}{|c|}{0.4500} \\
\hline & 10 & 23 & 36 & 49 & 10 & 23 & 36 & 49 & 10 & 23 & 36 & 49 & 10 & 23 & 36 & 49 \\
\hline Corn & 49.323 & 49.323 & 49.323 & 49.323 & 49.323 & 49.323 & 49.323 & 49.323 & 49.323 & 49.323 & 49.323 & 49.323 & 49.323 & 49.323 & 49.323 & 49.323 \\
\hline $\begin{array}{l}\text { Soybean meal } \\
45 \%\end{array}$ & 42.756 & 42.756 & 42.756 & 42.756 & 42.756 & 42.756 & 42.756 & 42.756 & 42.756 & 42.756 & 42.756 & 42.756 & 42.756 & 42.756 & 42.756 & 42.756 \\
\hline Soybean oil & 4.211 & 4.211 & 4.211 & 4.211 & 4.211 & 4.211 & 4.211 & 4.211 & 4.211 & 4.211 & 4.211 & 4.211 & 4.211 & 4.211 & 4.211 & 4.211 \\
\hline $\begin{array}{l}\text { Dicalciun } \\
\text { phosphate }\end{array}$ & 1.547 & 1.547 & 1.547 & 1.547 & 1.547 & 1.547 & 1.547 & 1.547 & 1.547 & 1.547 & 1.547 & 1.547 & 1.547 & 1.547 & 1.547 & 1.547 \\
\hline Limestone & 0.300 & 0.300 & 0.300 & 0.300 & 0.300 & 0.300 & 0.300 & 0.300 & 0.300 & 0.300 & 0.300 & 0.300 & 0.300 & 0.300 & 0.300 & 0.300 \\
\hline Dl-Methionine & 0.437 & 0.437 & 0.437 & 0.437 & 0.437 & 0.437 & 0.437 & 0.437 & 0.437 & 0.437 & 0.437 & 0.437 & 0.437 & 0.437 & 0.437 & 0.437 \\
\hline L-Lysine HCL & 0.335 & 0.335 & 0.335 & 0.335 & 0.335 & 0.335 & 0.335 & 0.335 & 0.335 & 0.335 & 0.335 & 0.335 & 0.335 & 0.335 & 0.335 & 0.335 \\
\hline L-Threonine & 0.024 & 0.024 & 0.024 & 0.024 & 0.024 & 0.024 & 0.024 & 0.024 & 0.024 & 0.024 & 0.024 & 0.024 & 0.024 & 0.024 & 0.024 & 0.024 \\
\hline Salt & 0.457 & 0.457 & 0.457 & 0.457 & 0.457 & 0.457 & 0.457 & 0.457 & 0.457 & 0.457 & 0.457 & 0.457 & 0.457 & 0.457 & 0.457 & 0.457 \\
\hline $\begin{array}{l}\text { Vit/min } \\
\text { suplement }\end{array}$ & 0.400 & 0.400 & 0.400 & 0.400 & 0.400 & 0.400 & 0.400 & 0.400 & 0.400 & 0.400 & 0.400 & 0.400 & 0.400 & 0.400 & 0.400 & 0.400 \\
\hline $\begin{array}{l}\text { Selenite blend } \\
45 \%\end{array}$ & 0.100 & 0.100 & 0.100 & 0.100 & 0.100 & 0.100 & 0.100 & 0.100 & 0.100 & 0.100 & 0.100 & 0.100 & 0.100 & 0.100 & 0.100 & 0.100 \\
\hline Vitamin E blend & 0.100 & 0.100 & 0.100 & 0.100 & 0.100 & 0.100 & 0.100 & 0.100 & 0.100 & 0.100 & 0.100 & 0.100 & 0.100 & 0.100 & 0.100 & 0.100 \\
\hline Antioxidant $^{2}$ & 0.010 & 0.010 & 0.010 & 0.010 & 0.010 & 0.010 & 0.010 & 0.010 & 0.010 & 0.010 & 0.010 & 0.010 & 0.010 & 0.010 & 0.010 & 0.010 \\
\hline Total & 100 & 100 & 100 & 100 & 100 & 100 & 100 & 100 & 100 & 100 & 100 & 100 & 100 & 100 & 100 & 100 \\
\hline
\end{tabular}


Table 4. Nutritional composition of the experimental diets for growing meat quails (14 to 35 days old) supplemented with different levels of inorganic selenium and vitamin $\mathrm{E}$

\begin{tabular}{|c|c|c|c|c|c|c|c|c|c|c|c|c|c|c|c|c|}
\hline \multirow{2}{*}{$\frac{\text { Selenium (mg/kg) }}{\text { Vitamin E (IU/kg) }}$} & \multicolumn{4}{|c|}{0.1125} & \multicolumn{4}{|c|}{0.2250} & \multicolumn{4}{|c|}{0.3375} & \multicolumn{4}{|c|}{0.4500} \\
\hline & 10 & 23 & 36 & 49 & 10 & 23 & 36 & 49 & 10 & 23 & 36 & 49 & 10 & 23 & 36 & 49 \\
\hline $\begin{array}{l}\text { Metabolizable energy } \\
(\mathrm{kcal} / \mathrm{kg})\end{array}$ & 3.036 & 3.036 & 3.036 & 3.036 & 3.036 & 3.036 & 3.036 & 3.036 & 3.036 & 3.036 & 3.036 & 3.036 & 3.036 & 3.036 & 3.036 & 3.036 \\
\hline $\begin{array}{l}\text { Available phosphorusl } \\
(\%)\end{array}$ & 0.41 & 0.41 & 0.41 & 0.41 & 0.41 & 0.41 & 0.41 & 0.41 & 0.41 & 0.41 & 0.41 & 0.41 & 0.41 & 0.41 & 0.41 & 0.41 \\
\hline Calcium (\%) & 0.61 & 0.61 & 0.61 & 0.61 & 0.61 & 0.61 & 0.61 & 0.61 & 0.61 & 0.61 & 0.61 & 0.61 & 0.61 & 0.61 & 0.61 & 0.61 \\
\hline Crude protein $(\%)$ & 23.5 & 23.5 & 23.5 & 23.5 & 23.5 & 23.5 & 23.5 & 23.5 & 23.5 & 23.5 & 23.5 & 23.5 & 23.5 & 23.5 & 23.5 & 23.5 \\
\hline Lysine digestible (\%) & 1.45 & 1.45 & 1.45 & 1.45 & 1.45 & 1.45 & 1.45 & 1.45 & 1.45 & 1.45 & 1.45 & 1.45 & 1.45 & 1.45 & 1.45 & 1.45 \\
\hline $\begin{array}{l}\text { Met.+cyst. digestible } \\
(\%)\end{array}$ & 1.04 & 1.04 & 1.04 & 1.04 & 1.04 & 1.04 & 1.04 & 1.04 & 1.04 & 1.04 & 1.04 & 1.04 & 1.04 & 1.04 & 1.04 & 1.04 \\
\hline $\begin{array}{l}\text { Threonine digestible } \\
(\%)\end{array}$ & 0.94 & 0.94 & 0.94 & 0.94 & 0.94 & 0.94 & 0.94 & 0.94 & 0.94 & 0.94 & 0.94 & 0.94 & 0.94 & 0.94 & 0.94 & 0.94 \\
\hline $\begin{array}{l}\text { Tryptophan digestible } \\
(\%)\end{array}$ & 0.29 & 0.29 & 0.29 & 0.29 & 0.29 & 0.29 & 0.29 & 0.29 & 0.29 & 0.29 & 0.29 & 0.29 & 0.29 & 0.29 & 0.29 & 0.29 \\
\hline Chlorine (\%) & 0.32 & 0.32 & 0.32 & 0.32 & 0.32 & 0.32 & 0.32 & 0.32 & 0.32 & 0.32 & 0.32 & 0.32 & 0.32 & 0.32 & 0.32 & 0.32 \\
\hline Sodium (\%) & 0.20 & 0.20 & 0.20 & 0.20 & 0.20 & 0.20 & 0.20 & 0.20 & 0.20 & 0.20 & 0.20 & 0.20 & 0.20 & 0.20 & 0.20 & 0.20 \\
\hline Potassium (\%) & 0.92 & 0.92 & 0.92 & 0.92 & 0.92 & 0.92 & 0.92 & 0.92 & 0.92 & 0.92 & 0.92 & 0.92 & 0.92 & 0.92 & 0.92 & 0.92 \\
\hline $\begin{array}{l}\text { Electrol. balance } \\
\mathrm{mEq} / \mathrm{kg}\end{array}$ & 232.69 & 232.69 & 232.69 & 232.69 & 232.69 & 232.69 & 232.69 & 232.69 & 232.69 & 232.69 & 232.69 & 232.69 & 232.69 & 232.69 & 232.69 & 232.69 \\
\hline
\end{tabular}


The hematocrit (HTC) was determined by microhematocrit methodology, using capillary tubes with ethylene diamine tetraacetic acid (EDTA). The tubes were centrifuged at $1200 \mathrm{rpm}$ for five minutes in a centrifuge (micro hematocrit centrifuge) and the percentage concentration of erythrocytes (red blood cells) was estimated using specific microhematocrit tables.

The viscera (heart, liver and gizzard) and lymphoid organs (spleen and bursa) of two birds per experimental unit (totaling six birds per treatment) that were slaughtered for blood collection at both ages were extracted via an incision in the abdominal cavity. The organs were weighed in analytical balance and their relative weights determined by means of the relation of the weight of the organ by the live weight of the bird multiplied by 100 .

The experimental data were statistically analyzed using SAS software (SAS Inst. Inc., Cary, NC, 2001), according to the model: $\mathrm{Yijkl}=\mathrm{b} 0+\mathrm{b} 1 \mathrm{Si}+\mathrm{b} 2 \mathrm{Vj}+\mathrm{b} 3 \mathrm{Si}$ $2+$ b4Vj $2+$ b5SVij $+F A+$ eijkl, where Yijkl was the variable measured in experimental unit $\mathrm{k}$, fed with a diet containing level $\mathrm{i}$ of $\mathrm{Se}$ and level $\mathrm{j}$ of $\mathrm{VE}$; b0 was the general constant and b1 was the coefficient of linear regression as a function of the level of inorganic $\mathrm{Se}$; $\mathrm{Si}$ was the Se level, S1=0.1125; $\mathrm{S} 2=0.2250 ; \mathrm{S} 3=0.3375$ and $\mathrm{S} 4=0.4500$ $(\mathrm{mg} / \mathrm{kg}) ; \mathrm{Vj}$ was the VE level, $\mathrm{V} 1=10$; $\mathrm{V} 2=23 ; \mathrm{V} 3=36$ and $\mathrm{V} 4=49$ (IU/kg); b2 was the linear regression coefficient as a function of VE level; b3 was the coefficient of quadratic regression as a function of the level of inorganic Se; b4 was the quadratic regression coefficient as a function of VE level; b5 was the coefficient of linear regression as a function of the interaction between the level of Se and level of VE; FA was the lack of adjustment of the regression model; Eijkl was the random error associated with each observation.

\section{RESULTS}

\section{Viscera biometry}

At 14 days of age, the relative weights of the heart $(\mathrm{P}=0.0169)$ and spleen $(\mathrm{P}=0.0203)$ showed a respective reduction and linear increase as a function of the VE level (Table 5). At 35 days of age, the relative weights of the liver, gizzard and spleen were not influenced by the levels of Se and VE $(\mathrm{P}>0.05)$. The relative weights of the bursa $(\mathrm{P}=0.0390)$ and heart $(\mathrm{P}=0.0357)$ showed a respective increase and a linear reduction as a function of the $\mathrm{Se}$ level (Table 6).

Blood parameters

At 14 days of age, an effect on TC concentration was verified as a function of the interaction $(\mathrm{P}=0.0097)$ between the Se and VE levels. (Table 7). The concentrations were also influenced by a quadratic effect $(\mathrm{P}=0.0138)$ related to $\mathrm{Se}$ levels and a linear reduction $(\mathrm{P}=0.0275)$ as a function of increasing VE. The minimum TC concentration of $124.19 \mathrm{mg} / \mathrm{dL}$ was obtained using 0.33 $\mathrm{mg} \mathrm{Se} / \mathrm{kg}$ of feed.

The ALT enzyme was influenced $(\mathrm{P}=0.000)$ by a quadratic function of the VE level. The estimate of $24.52 \mathrm{U} / \mathrm{L}$ for the maximum ALT activity was determined at a level of $31.31 \mathrm{IU}$ of $\mathrm{VE} / \mathrm{kg}$ of feed. The \% HTC showed similar behavior to the TC concentration, with a quadratic effect for $\mathrm{Se}(\mathrm{P}=0.0404)$ and a linear reduction $(\mathrm{P}=0.0038)$ with increasing $\mathrm{VE}$ in the diet. The estimate of $27.35 \%$ for the lowest HTC percentage was determined with $0.27 \mathrm{mg} \mathrm{Se} / \mathrm{kg}$ in the feed. 
Table 5. Relative weight of viscera and lymphoid organs of meat quails at 14 days of age supplemented with different levels of inorgar selenium and vitamin $\mathrm{E}$

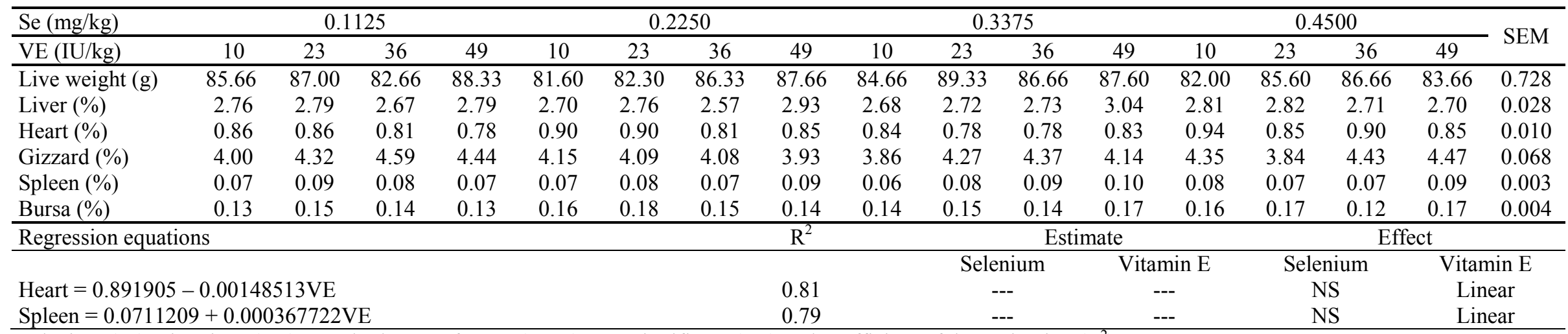

Selenium (Se); vitamin E (VE); standard error of mean (SEM); non-significant (NS) and coefficient of determination ( ${ }^{2}$ )

Table 6. Relative weight of viscera and lymphoid organs of meat quails at 35 days of age supplemented with different levels of inorganic selenium and vitamin $\mathrm{E}$

\begin{tabular}{|c|c|c|c|c|c|c|c|c|c|c|c|c|c|c|c|c|c|}
\hline \multirow{2}{*}{$\begin{array}{l}\mathrm{Se}(\mathrm{mg} / \mathrm{kg}) \\
\mathrm{VE}(\mathrm{IU} / \mathrm{kg})\end{array}$} & \multicolumn{4}{|c|}{0.1125} & \multicolumn{4}{|c|}{0.2250} & \multicolumn{4}{|c|}{0.3375} & \multicolumn{4}{|c|}{0.4500} & \multirow{2}{*}{ SEM } \\
\hline & 10 & 23 & 36 & 49 & 10 & 23 & 36 & 49 & 10 & 23 & 36 & 49 & 10 & 23 & 36 & 49 & \\
\hline Live weight (g) & 226.7 & 215.8 & 222.5 & 240.0 & 230.0 & 227.5 & 235.0 & 229.2 & 230.8 & 225.8 & 238.3 & 225.8 & 226.7 & 230.8 & 228.3 & 219.2 & 1.301 \\
\hline Liver (\%) & 2.06 & 2.10 & 1.76 & 1.94 & 2.13 & 2.12 & 2.02 & 2.11 & 1.97 & 1.71 & 1.82 & 1.93 & 2.05 & 2.19 & 1.97 & 1.93 & 0.045 \\
\hline Heart (\%) & 0.83 & 0.85 & 0.86 & 0.81 & 0.84 & 0.82 & 0.86 & 0.80 & 0.81 & 0.84 & 0.87 & 0.82 & 0.80 & 0.82 & 0.74 & 0.80 & 0.003 \\
\hline Gizzard (\%) & 2.10 & 1.68 & 1.82 & 1.83 & 1.85 & 1.99 & 1.82 & 1.79 & 1.69 & 1.89 & 1.85 & 1.95 & 1.94 & 1.95 & 1.95 & 1.73 & 0.003 \\
\hline Spleen (\%) & 0.07 & 0.06 & 0.07 & 0.08 & 0.07 & 0.09 & 0.07 & 0.06 & 0.08 & 0.09 & 0.07 & 0.07 & 0.09 & 0.09 & 0.07 & 0.07 & 0.003 \\
\hline Bursa (\%) & 0.11 & 0.12 & 0.13 & 0.12 & 0.12 & 0.13 & 0.14 & 0.11 & 0.15 & 0.14 & 0.14 & 0.14 & 0.14 & 0.14 & 0.14 & 0.14 & 0.004 \\
\hline \multicolumn{5}{|c|}{ Regression equations } & & & & $\mathrm{R}^{2}$ & & \multicolumn{4}{|c|}{ Estimate } & \multicolumn{4}{|c|}{ Effect } \\
\hline \multicolumn{5}{|c|}{$\begin{array}{l}\text { Heart }=0.862512-0.129690 \mathrm{Se} \\
\text { Bursa }=0.119689+0.0561344 \mathrm{Se}\end{array}$} & & & & $\begin{array}{l}0.61 \\
0.77\end{array}$ & & Sel & $\begin{array}{l}\text { lium } \\
-\end{array}$ & \multicolumn{2}{|c|}{ Vitamin E } & \multicolumn{2}{|c|}{$\begin{array}{l}\text { Selenium } \\
\text { Linear }\end{array}$} & \multicolumn{2}{|c|}{$\begin{array}{l}\text { Vitamin E } \\
\text { NS }\end{array}$} \\
\hline
\end{tabular}

Selenium (Se); vitamin E (VE); standard error of mean (SEM); non-significant (NS) and coefficient of determination ( ${ }^{2}$ ). 
Table 7. Blood parameters of meat quails at 14 days of age supplemented with different levels of inorganic selenium and vitamin $\mathrm{E}$

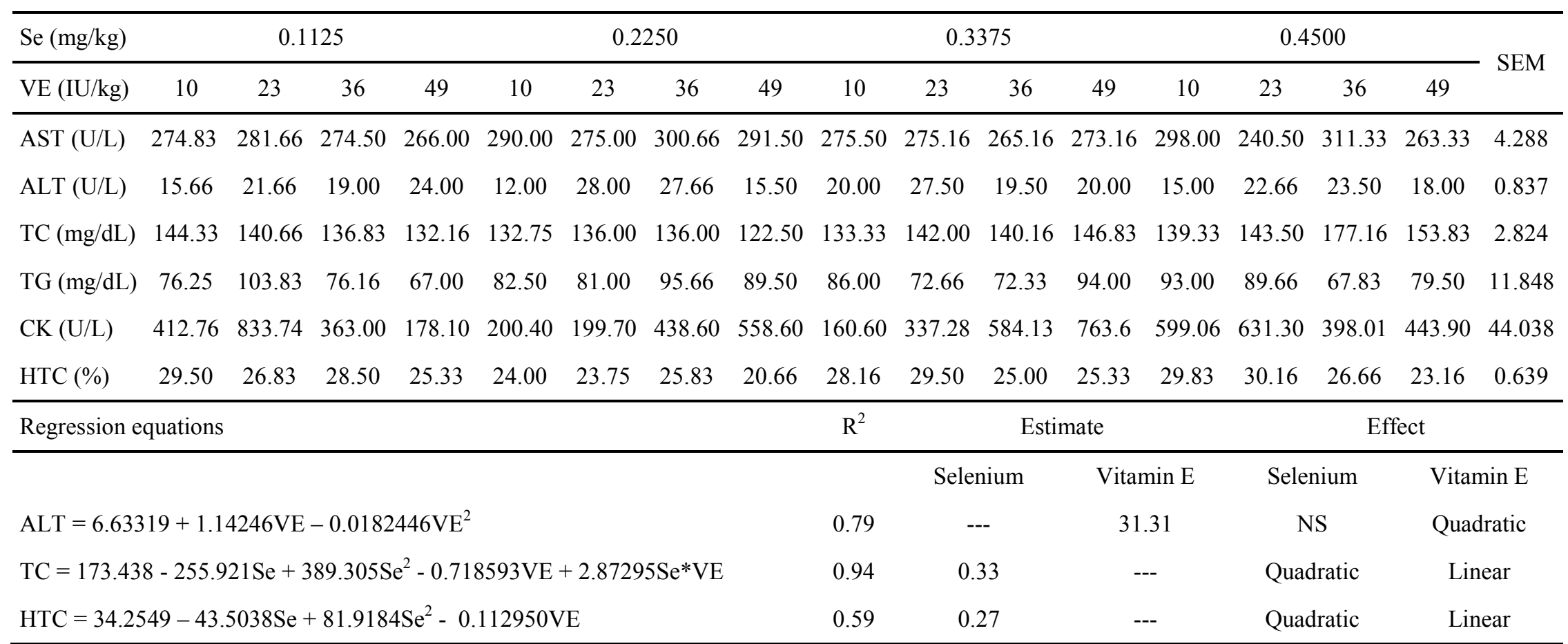

Selenium (Se); vitamin E (VE); standard error of mean (SEM); aspartate aminotransferase (AST); alanine aminotransferase (ALT); total cholesterol (TC); triglycerides (TG); creatine quinase (CK); hematocrits (HTC); non-significant (NS) and coefficient of determination $\left(\mathrm{R}^{2}\right)$ 
In the blood evaluations at 35 days of age, the TC $(\mathrm{P}=0.0055)$ and $\mathrm{TG}$ concentrations $(\mathrm{P}=0.0220)$ showed a quadratic effect as a function the $\mathrm{Se}$ level (Table 8). The minimum concentrations of TC $(175.23 \mathrm{mg} / \mathrm{dL})$ and TG $(104.63 \mathrm{mg} / \mathrm{dL})$ were determined with estimated Se levels of 0.26 and $0.29 \mathrm{mg} / \mathrm{kg} /$ feed respectively. The CK enzyme increased linearly as a function of the Se level $(\mathrm{P}=0.0478)$.

\section{DISCUSSION}

The increase in the relative weight observed for the spleen at 14 days of age, despite being influenced only by the VE levels, was expected as a result of the influence that VE exerts on the immune system (Table 5). The spleen is a secondary lymphoid organ and its main function is to promote the removal of circulating antigenic particles and aged erythrocytes (SILVA, 2009). At 35 days of age, a linear increase in bursa weight was also observed (Table 6). The increase in the relative weights of both organs correlated with increased lymphocyte activity, which provides an increase in antibody production in birds (Scott, 2004). In the same sense, Ribeiro et al. (2008) reported that the size and relative weight of the bursa are characteristics that may serve as indicators of stress, which can trigger reductions in the size of lymphoid organs.

Se supplementation in the diets decreased the relative weight of the heart. According to Khan et al. (1993), excess $\mathrm{Se}$ in feed can generate intoxication and cause an increase in the relative weight of the heart, but this did not occur in our experiment, probably because the levels used in the rations in this study are low and incapable of generating intoxication in birds. According to the NRC (1994), safe levels that do not result in deficiency or toxicity of Se in birds lie between 0.15 and $4.0 \mathrm{mg} \mathrm{Se} / \mathrm{kg}$ of feed. Upton et al. (2008) did not verify an increase in the percentage of viscera of broiler chickens when they tested diets with 0 and $0.2 \mathrm{ppm}$ sodium selenite in the diet. They reported that increased viscera weight may be the result of slower feed passage, resulting in greater intestinal feed retention, thus making food utilization more efficient. At 14 days of age, an interaction between Se and VE was observed, demonstrating that they acted together in the determination of serum TC levels (Table 7). Increasing levels of provided $\mathrm{Se}$ allowed the estimation of the lowest TC concentration point $(124.19 \mathrm{mg} / \mathrm{dL})$, while increasing VE levels also caused TC levels to decrease linearly (Figure 1). According to Kang et al. (2000), when the deposition of cholesterol exceeds its clearance in the arterial walls, diseases begin to emerge. The same authors performed a study with rabbits where they found that the addition of $1 \mathrm{ppm}$ sodium selenite in a high fat diet resulted in a decrease in TC and TG levels compared with the same diet without the addition of the mineral $(\mathrm{P}<0.01)$. They further concluded that the Se has a hypocholesterolemic effect. Regarding the main aminotransferase enzymes, AST or glutamic oxalacetic transaminase (GOT) is present both in the cytosol and in the mitochondria. In addition, it is at high concentrations in many tissues, such as heart, liver, skeletal muscle, kidneys and pancreas. ALT or pyruvic glutamic transaminase (PGT) has a more specific distribution, being limited to the cytosol in hepatocytes. 
Table 8. Blood parameters of meat quails at 35 days of age supplemented with different levels of inorganic selenium and vitamin $\mathrm{E}$

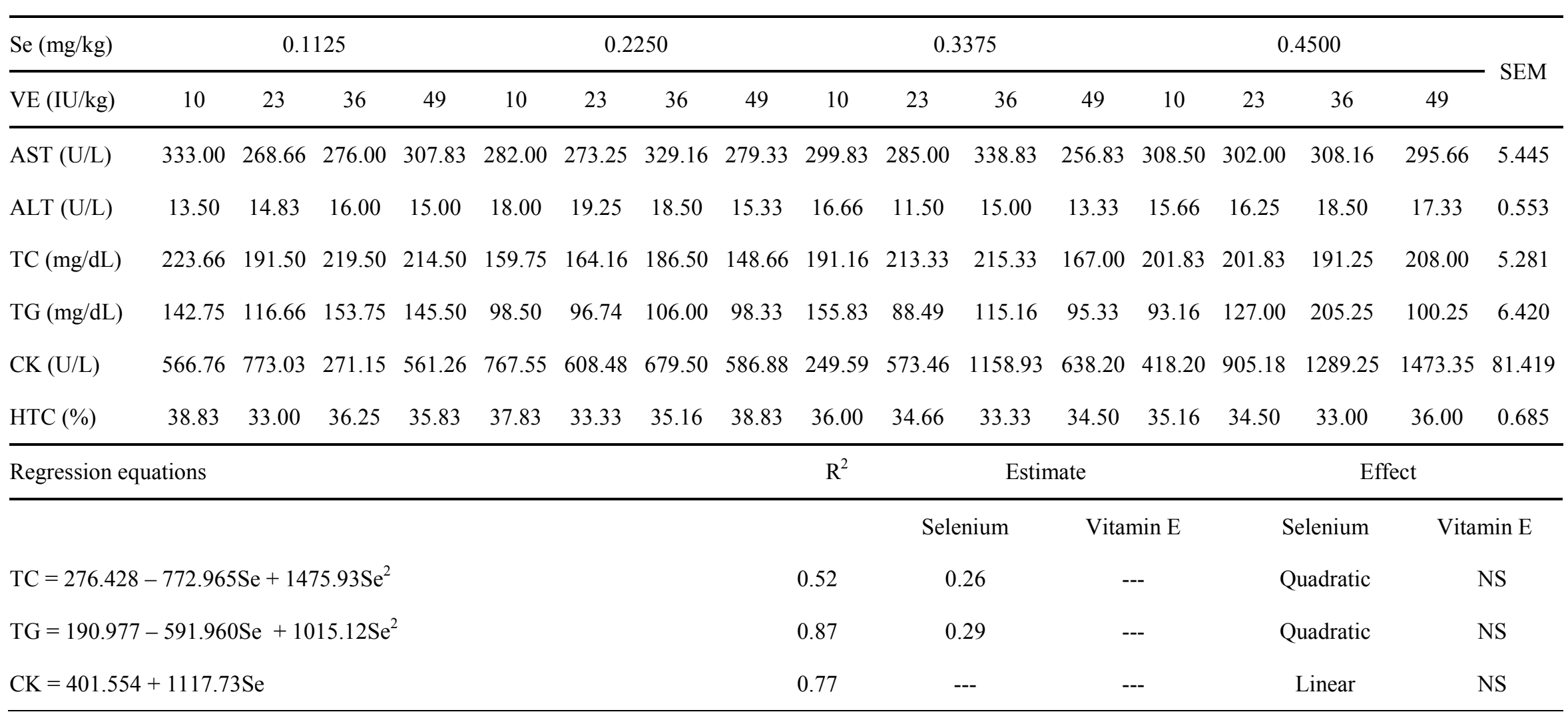

Selenium (Se); vitamin E (VE); standard error of mean (SEM); aspartate aminotransferase (AST); alanine aminotransferase (ALT); total cholesterol (TC); triglycerides (TG); creatine quinase (CK); hematocrits (HTC). non-significant (NS) and coefficient of determination $\left(\mathrm{R}^{2}\right)$ 


$$
\begin{gathered}
\mathrm{TC}=173.438-255.921 \mathrm{Se}+389.305 \mathrm{Se}^{2}-0.18593 \mathrm{VE} \\
\mathrm{R}^{2}=0.94
\end{gathered}
$$

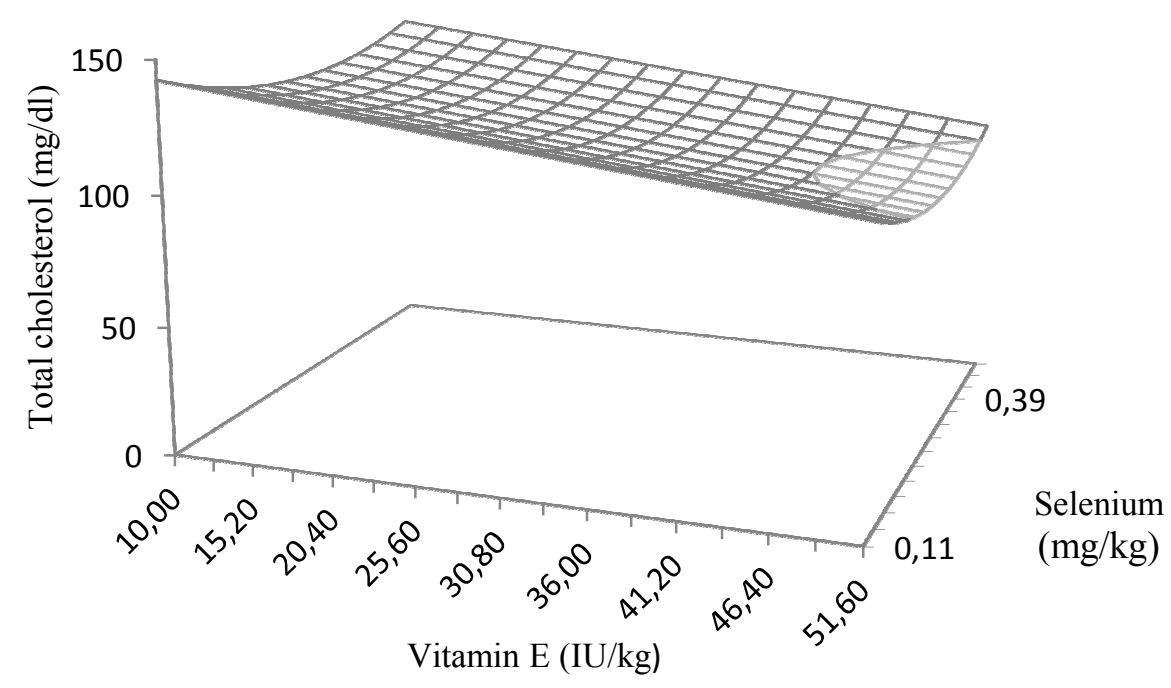

Figure 1. Total cholesterol levels for meat quails at 14 days of age in function of inorganic selenium and vitamin $\mathrm{E}$ levels in diets

According to reports by Lumeij \& Westerhof (1987), changes in the serum concentrations of liver enzymes are not always related to pathological changes in the liver of birds. The peak ALT concentration $(24.52 \mathrm{U} / \mathrm{L})$ may be related, for example, with the occurrence of hemolysis, which may be reflected in variations in the results (Table 8). ALT is found both in hepatocytes and in the muscles and other tissues of birds, so measurements of this enzyme are limited in their ability to predict liver damage in birds (SCHMIDT et al., 2007a). In their studies, Borsa et al. (2006) suggest levels of 9 to $22 \mathrm{U} / \mathrm{L}$ for serum concentrations of ALT in broilers at 14 days of age; these are similar values to those found for the meat quails in this experiment. The authors also reported that there are variations in the results found by other authors, and that several factors such as the commercial kits used, the methodology used to obtain the samples and the apparatus used to measure dosages may cause changes in enzymatic serum levels.

The hematocrit is one of the parameters used to determine the degree of anemia, corresponding to the percentage of red blood cells as a function of the total volume of blood, and its results may suggest hematological disturbances (MAXWELL et al., 1992). In the literature, reduction of hematocrit values suggests anemia in birds, but in this study, although a linear reduction in the hematocrit was obtained, this is not believed to be due to anemia because the bursa weights increased linearly as a function of the levels of $\mathrm{Se}$ in the diet (Table 8). Schmidt et al. (2007b), suggest that the hematocrit can vary according to the age and the sex of birds. The enzyme $\mathrm{CK}$ is characteristic of muscle tissues, and is released in cases of lesions or dystrophy (STOYANCHEV, 2007). Some studies have shown that genetically enhanced 
strains for rapid growth and muscle hypertrophy have $\mathrm{CK}$ activity that is exaggeratedly increased with age. These observations have also been verified in broilers (SZABÓ \& MILISITS, 2007).

The results of the obtained for serum CK levels at 35 days of age in this study were inconclusive. The higher levels of Selenium should provide lower serum concentrations of the enzyme in the birds, but exactly the opposite occurred. In addition, such large concentration variations were not expected, as the species used has not been improved for rapid growth or muscle hypertrophy.

The linear increases in spleen weight and bursa at 14 and 35 days of age, respectively, suggest an improvement in the immune response of the birds as a result of vitamin $\mathrm{E}$ and selenium supplementation. To obtain the minimum concentrations of TC and TG in the blood at 35 days of age, supplementation of 0.26 and 0.29 $\mathrm{mg} / \mathrm{Se} / \mathrm{kg} /$ feed is recommended.

\section{REFERÊNCIAS}

ALBUQUERQUE, D.M.N.; LOPES, J.B.; FERRAZ, M.S.; RIBEIRO, M.N; SILVA, S.R.G.; COSTA, E.M.S.; LIMA, D.C.P.; FERREIRA, J.D.M.; GOMES, P.E.B.; LOPES, J.C.O.

Vitamin $\mathrm{E}$ and organic selenium for broilers from 22 to 42 days old: performance and carcass traits. Anais da Academia Brasileira de Ciências, v.89, p.1259-1268, 2017.

ARSLAN, M.; ÖZCAN, M.; MATUR, E.; ÇÖTELOGLU, Ü.; ERGÜL, E. The effects of vitamin $E$ on some blood parameters in broilers. Turkish Journal of Veterinay and Animal Sciences, v.25, p.711-716, 2001.
BORSA, A.; KOHAYAGAWA, A.; BORETTI, L.P.; SAITO, M.E.;

KUIBIDA, K. Níveis séricos de enzimas de função hepática em frangos de corte de criação industrial clinicamente saudáveis.

Arquivo Brasileiro de Medicina

Veterinária e Zootecnia, v.58, p.675677, 2006.

CHEN, G., WU, J., CHONG, L. The effect of different selenium levels on production performance and biochemical parameters of broilers. Italian Journal of Animal Science, v.12, p. 486-491, 2013.

DE ALMEIDA, J.N., DOS SANTOS, G.R., BETETO, F.M.; MEDEIROS, L.G.de; OBA, A.; SHIMOKOMAKI, M.; SOARES, A.L. Dietary supplementation of chelated selenium and broiler chicken meat quality. Semina-Ciencias Agrarias, v.33, p.3117-3122, 2012.

HABIBIAN, M., GHAZI, S., MOEINI, M.M.; ABDOLMOHAMMADI, A. Effects of dietary selenium and vitamin $\mathrm{E}$ on immune response and biological blood parameters of broilers reared under thermoneutral or heat stress conditions.

International Journal of

Biometeorology, v.57, p.741-752, 2013.

HABIBIAN, M.; GHAZI, S.; MOEINI, M.M. Effects of dietary selenium and vitamin $\mathrm{E}$ on growth performance, meat yield, and Selenium content and lipid oxidation of breast meat of broilers reared under heat stress. Biology Trace

Element Research, v.169, p.142-152, 2015.

KANG, B.P.S.; BANSAL, M.P.; MEHTA, U. Hyperlipidemia and type I 5'-monodeiodinase activity: regulation by selenium supplementation in rabbits.

Biological Trace Element Research. v.7, p.231-239, 2000. 
KHAN, M.Z.; SZEREK, J.;

MARKIEWICZ, K. Effects of oral administration of toxic levels of lead and selenium upon concentration of different elements in the liver of broiler chicks.

Journal of Veterinary Medicine, v.40, p.652-664, 1993.

LUMEIJ, J.T. WESTERHOF. Blood chemistry for the diagnosis of hepatobiliary disease in birds. A review. Veterinary Quaterly, v.9, p.255-61, 1987.

MAXWELL, M.H.; ROBERTSON, G.W.; MCCORQUODALE, C.C. Whole blood and plasma viscosity in normal and 28reqüên broiler chickens. British Poultry Science, v.33, p.871-877, 1992.

\section{NATIONAL RESEARCH COUNCIL - NRC. Nutrient requirements of poultry. 9th revised ed. Washington: National Academy Press, 1994.}

RIBEIRO, A.M.L., VOGT, L.K., CANAL, C.W.; LAGANÁ, C.; STRECK, A.F. Suplementação de vitaminas e minerais orgânicos e sua ação sobre a imunocompetência de frangos de corte submetidos a estresse por calor.

Revista Brasileira de Zootecnia, v.37, p.636-644, 2008.

ROSTAGNO, H.S., ALBINO, L.F.T., DONZELE, J.L.; GOMES, P.C.; OLIVEIRA, R.F.de; LOPES, D.C.; FERREIRA, A.S.; BARRETO, S.L. de T.; EUCLIDES, R.F. Tabelas brasileiras para aves e suínos: composição de alimentos e exigências nutricionais. 3.ed. Viçosa: Universidade Federal de Viçosa, 2011.

SCHMIDT, E.M.S.; LOCATELLIDITTRICH, R.; SANTIN, R.; PAULILLO, A.C. Patologia clínica em aves de produção - Uma ferramenta para monitorar a sanidade avícola - Revisão. Archives of Veterinary Science, v.12, p.9-20, 2007a.
SCHMIDT, E.M.S.; PAULILLO, A.C.; SANTIN, E.; DITTRICH, R.L.;

OLIVEIRA, E.G de. Hematological and serum chemistry values for the ringnecked pheasant (Phasianus colchicus): variation with sex and age.

International Journal of Poultry

Science, v.6, n.2, p.137-139, $2007 b$.

SCOTT, T.R.. Our current understanding of humoral immunity of poultry. Poultry Science, v.82, p.15591564, 2004.

ŠEVČÍKOVÁ, S.; SKŘIVAN, M.; DLOUHÁ, G.; KOUCKÝ, M. The effect of selenium source on the performance and meat quality of broiler chickens. Czech Journal of Animal Science, v.51, p.449-457, 2006.

SILVA, I.C.M. Resposta imune e desempenho de frangos de corte submetidos a variações dietéticas de vitamina E e selênio. 2009. 163f. Tese (Doutorado em Zootecnia) Universidade Federal do Rio Grande do Sul, Porto Alegre.

SILVA, R.M.; FURLAN, A.C.; TON, A.P.S.; MARTINS, E.N.; SCHERER, C.; MURAKAMI, A.E. Exigências nutricionais de cálcio e fósforo de codornas de corte em crescimento.

Revista Brasileira de Zootecnia, v.38, p.1509-1524, 2009.

STOYANCHEV, K. Effects on the environmental stress on experimentally induced muscular dystrophy in broiler turkeys. Revue Médécine Vétérinaire, v.58, p.190-195, 2007.

SURAI, P.F.; SPARKS, N.H.C.; SPEAK, B.K. The role of antioxidants in reproduction and fertility of poultry. In: EUROPEAN POULTRY CONFERENCE, 12., 2006, Verona, Italy. Proccengins... Verona, Italy, 2006. 
SZABÓ, A., ILISITS, G.M.

Clinicochemical follow-up of broiler rearing- a five - week study. Acta Veterinaria Hungarica, v.55, p.451462, 2007.

SUHAJDA, A.; HEGÓCZKI, J.;

JANZSÓ, B.; PAIS, I, VERECZKEY, G. Preparation of selenium yeasts I. Preparation of selenium-enriched Saccharomyles cerevisiae. Journal of Trace Elements in Medicine and Biology, v.14, p.43-47, 2000.

THRALL, M.A. Veterinary Hematology and Clinical Chemistry. Philadelphia, Lippincott: Williams \& Wilkins, 2004. 518p.

TOLEDO, G.S.; KLOECKNER, P.; LOPES, J.; COSTA, P.T. Níveis das vitaminas $\mathrm{A}$ e $\mathrm{E}$ em dietas de frangos de corte de 1 a 42 dias de idade. Ciência Rural, v.36, p.624-629, 2006.

UPTON, J.R.; EDENS, F.W.; FERKET, P.R. Selenium yeast effect on broiler performance. International Journal of Poultry Science, v.7, p.798-805, 2008.

VALLE, S.F.; ALLGAYER, M.C.; PEREIRA, R.A.; BARCELLOS, L.J.G.; HLAVAC, N.R.C.; FRANÇA, R.T.; LOCATELLI, M.L. Parâmetros de bioquímica sérica de machos, fêmeas e filhotes de Araras Canindé (Ara ararauna) saudáveis mantidas em cativeiro comercial. Ciencia Rural, v.38, p.711-716, 2008.

Data de recebimento: 12/07/2016

Data de aprovação: 16/08/2017 